\title{
Health Status of Elder Spinal Cord Veterans
}

\section{ART I C L E INF O}

\section{Article Type}

Descriptive Study

\section{Authors}

Javadi Parvaneh M.* $M D, M P H$

Sahaf R. ${ }^{1} P h D$

Delbari A. ${ }^{1} P h D$,

Kamrani A.A. ${ }^{2} M D$

Fadaye Vatan R. ${ }^{2} P h D$

\section{How to cite this article} Javadi Parvaneh M,SahafR, Delbari A, Kamrani A A, Fadaye Vatan R. Health Status of Elder Spinal Cord Veterans. Iranian Journal of War \&Public Health.2017:9(2):97-103.

\section{A B S T R A C T}

Aims Noticing the veteran's old age, and especially veterans with spinal-cord injuries who constitute most of the veterans with physical-motor problems, it is necessary to assess their health. The aim of the study was to investigate comprehensively the health statuses of veterans with spinal-cord injuries in their higher than threescore.

Instrument \& Methods In the descriptive cross-sectional study, 53 veterans with spinal-cord injuries in their higher than threescore, who participated in a therapeutic recreation camp from all the provinces, were studied in 2011. The subjects were selected via census method. Collected by questionnaires, data of their nutritional status, bedsores risk, cognition status, depression level, and daily functioning with the utilization of instruments, as well as need and dependency in daily activities was analyzed by SPSS 14 software using descriptive statistics and Chi-square and Mann-Whitney tests.

Findings 32, 27, and 47 persons were with depression symptoms, malnutrition risk, and bedsores risk, respectively. 47 persons were with either no or mild cognitive impairment. 35 persons were independent in the instrumental activities in daily living (IADL). Only 10 persons were with mild or minimum dependency in daily life. The nutrition status significantly correlated with depression factors $(\mathrm{p}<0.001)$, IADL $(\mathrm{p}=0.04)$, and dependency level in daily life $(\mathrm{p}=0.038)$.

Conclusion Most of the elderly veterans with spinal-cord injuries are without any cognitive impairment, with depression symptoms, and with malnutrition risk, as well as bedsores risk. In addition, they are dependent in their daily activities, while independent in their daily life using instrumental aids.

Keywords Elderly; Aging; Health, Veterans; Spinal Cord Injury

\section{I T A T I O N L I N KS}

[1] Health and wellbeing for older people ... [2] General Census of Population and Housing ... [3] Health status of iranian older people ... [4] GComprehensive health assessment of senior citizens ... [5] Demographic of aging in Geriatric ... [6] Significance of an aging-linked neuron binding ... [7] To investigate the relationship between cognitive ... [8] The MERCK manual of ... [9] Levels of disability among the elderly in institutionalized and home-based ... [10] Aging and mental health: Positive psychosocial and biomedical ... [11] Mental health promotion in older adults: Addressing ... [12] International differences in ageing and spinal ... [13] Adjustment in the family and patterns of family ... [14] A review and conceptual re-examination of mental toughness: Implications for future ... [15] Assessing the nutritional status of the elderly: The Mini Nutritional Assessment as part of the geriatric ... [16] Nutritional status using multidimensional assessment in elderly ... [17] A behavioural genetic study of ... [18] The reliability and validity of the mini-mental state examination [19] Validity of the farsi version of mini-mental ... [20] Development and validation of a geriatric depression screening ... [21] Reliability, validity and factor structure of the CES-D in Iranian [22] Assessment of older people: Self-monitoring and ... [23] Validity and reliability of the persian version of lawton instrumental activities ... [24] Physical... [25] The elderly population in Iran: An evergrowing concern ... [26] Nutrition and aging: changes in the regulation ... [27] Medication use as a risk factor for falls ... [28] Hazzard's geriatric medicine and ... [29] Assessment of health status of elderly people ... [30] Health maintenance of the ... [31] Comprehensive geriatric assessment: A meta-analysis of controlled ... [32] Multiple losses in older ... [33] Geriatric depression scale (GDS) recent evidence and development ... [34] Frail elderly as disaster victims: Emergency management

\section{Article History}

Received: October 2, 2016

Accepted: February 25, 2017

ePublished: April 24, 2017 


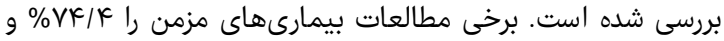

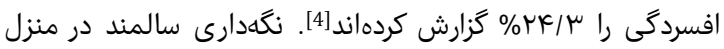

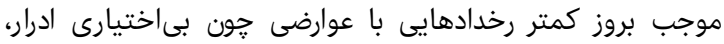

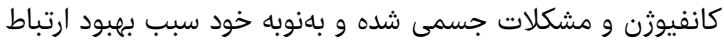

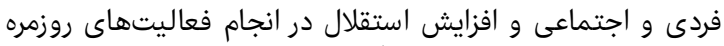

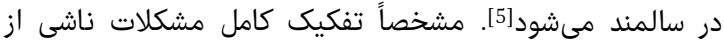

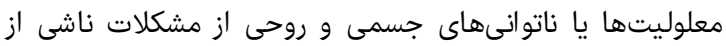

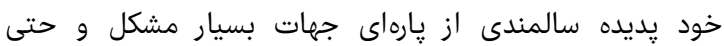

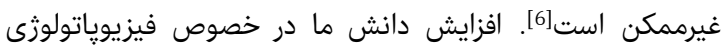

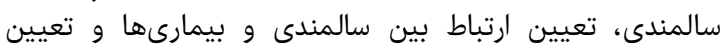

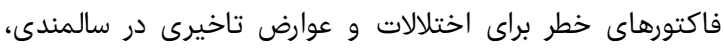

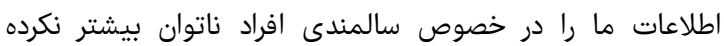

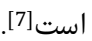

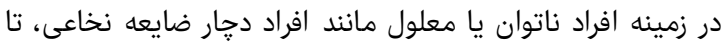

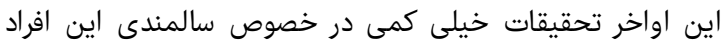

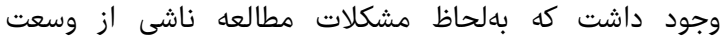

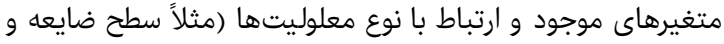

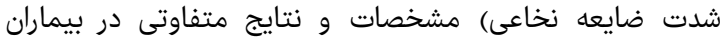

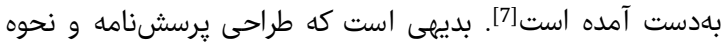

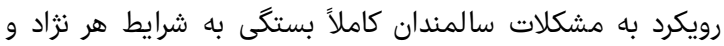

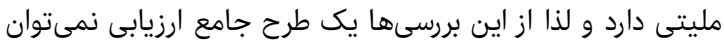

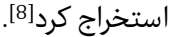

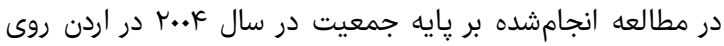

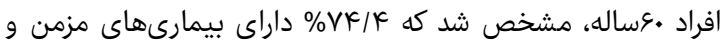

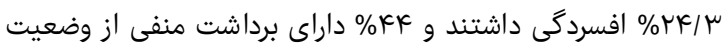

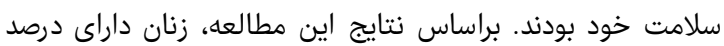

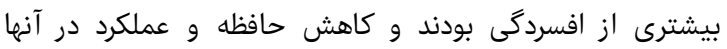

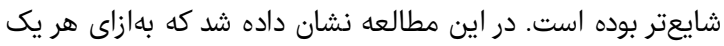

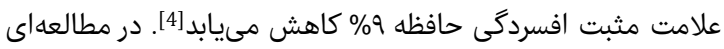

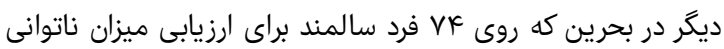

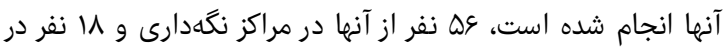

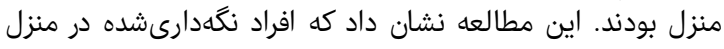

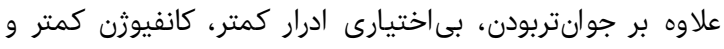

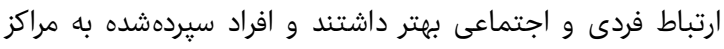

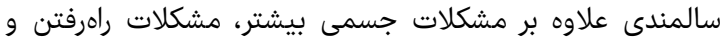

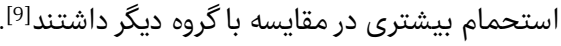

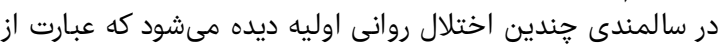

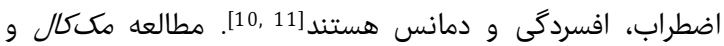

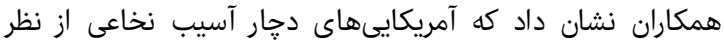

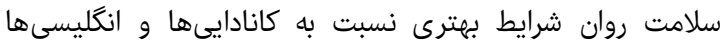

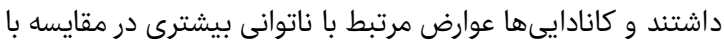

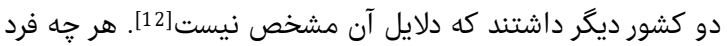

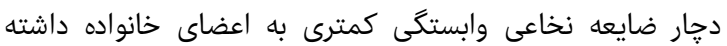

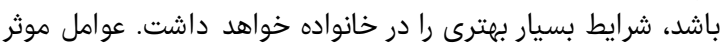

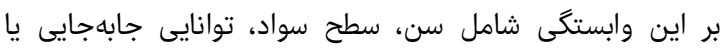

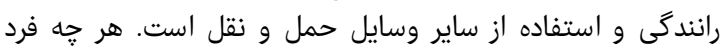

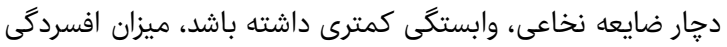

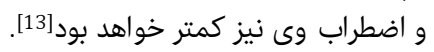

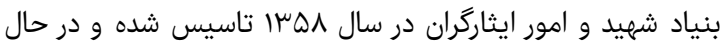

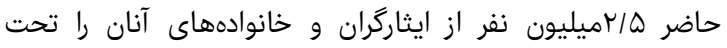

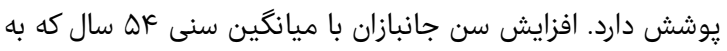

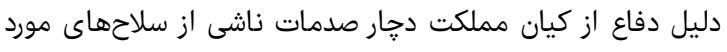

دوره 9، شماره r، بهار عوسا
وضعيت سلامت جانبازان نخاعى سالمند

\author{
MD, MPH "مسعود جوادى يروانه

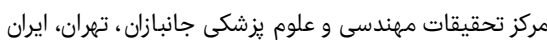

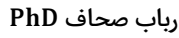 \\ مركز تحقيقات سالمندى، دانشكاه علوم بهزيستى و توانبخشى، تهران، ايران \\ احمد دلبرى PhD \\ مركز تحقيقات سالمندى ، دانشكاه علوم بهزيستى و توانبخشى، تهران، ايران

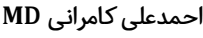 \\ "مركز تحقيقات سالمندى" و و "گروهانى سالمندى"، دانشكاه علوم بهزيستى و \\ توانبخشى، تهران، ايران

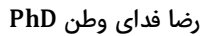

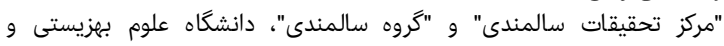

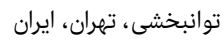

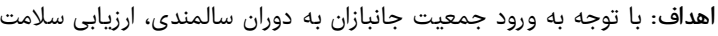

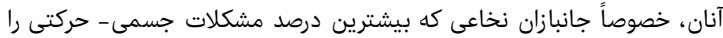

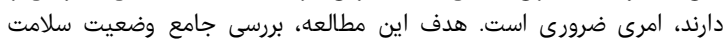

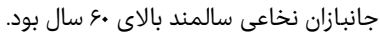

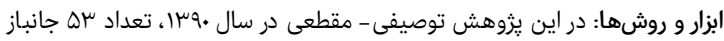

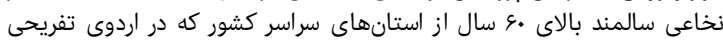

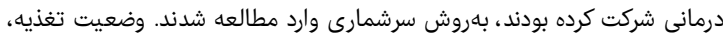

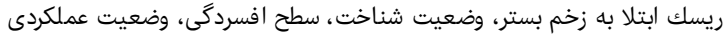

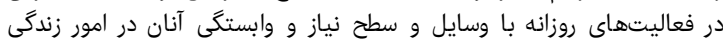

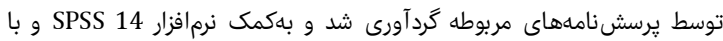

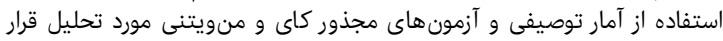

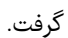

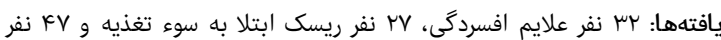

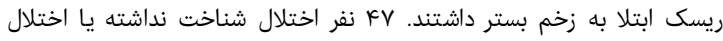

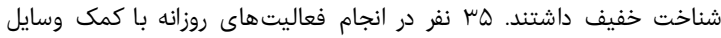

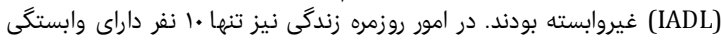

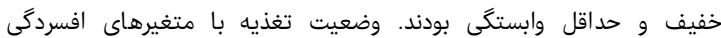

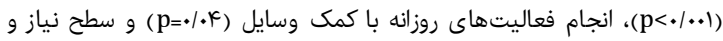

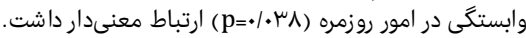

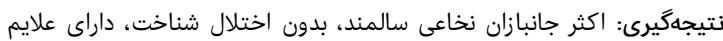

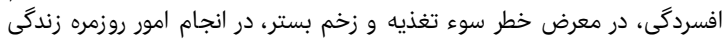

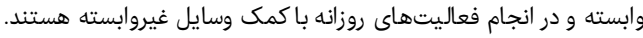
كليدوازمها: سالمند، سالمندى، سلامت، جانباز، آسيب نخاعى رائ

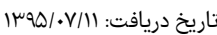

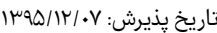

masoudjavadi@gmail.com : تويسنده مسئول:

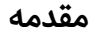

مساله جمعيت رو به افزايش سالمندان توجه سياست گزاران را به آنه

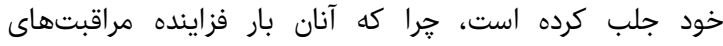

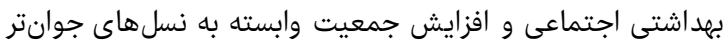

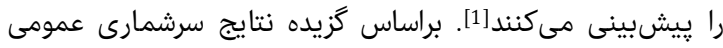

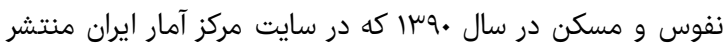
شده، جمعيت كل ايران

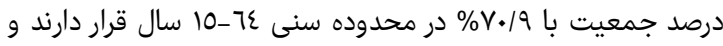

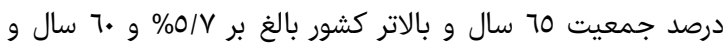

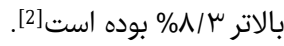

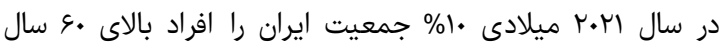

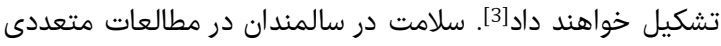

فصل نامه علمى - يخروهشى طب جانباز 
وضعيت سلامت جانبازان نخاعى سالمند

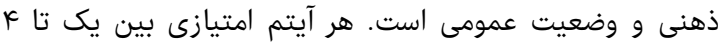

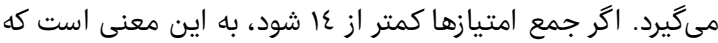

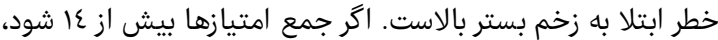

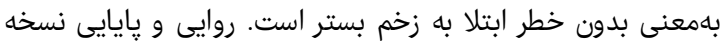

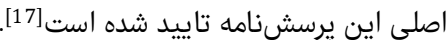

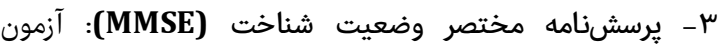
بيشتر از همه، در سالمندان براى بررسى اختى اختلال شناختى

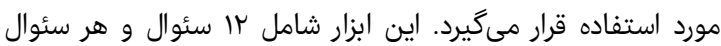

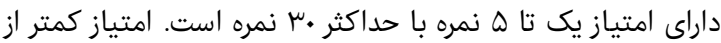

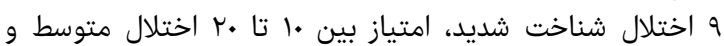

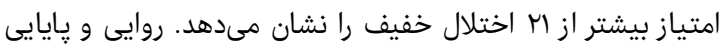

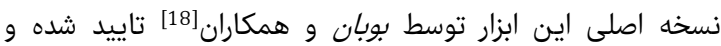

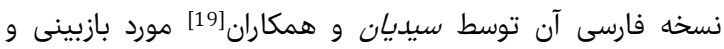
ترجمه قرار گرفته است فارسي

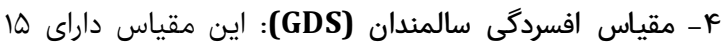

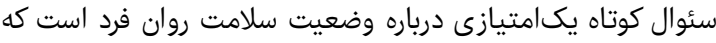

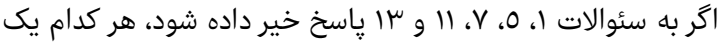

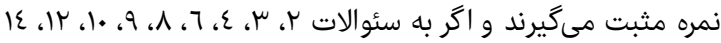

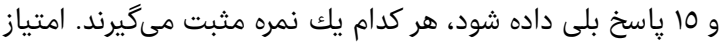

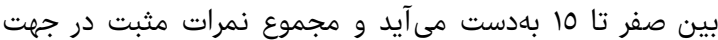

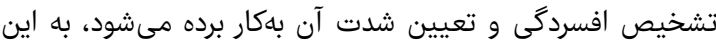

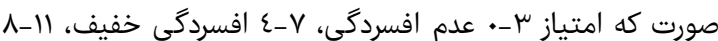

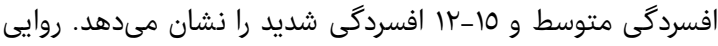

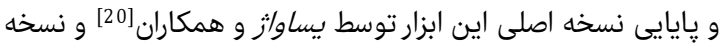

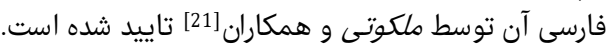

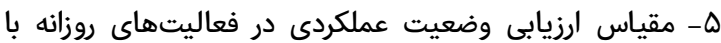

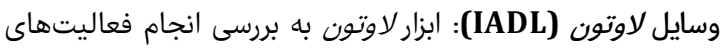

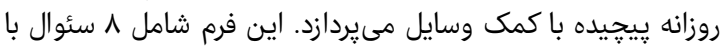

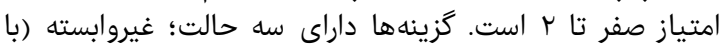

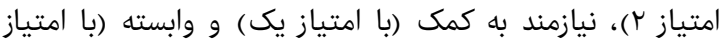

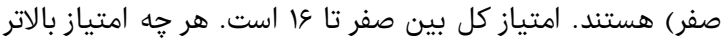

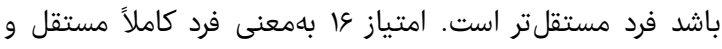

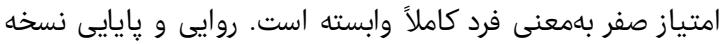

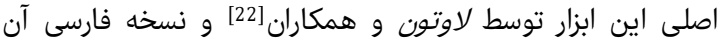

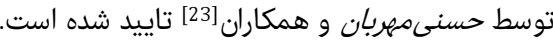

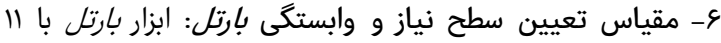

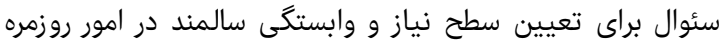

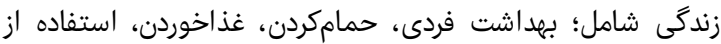

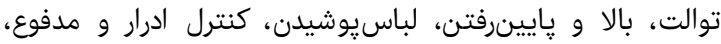

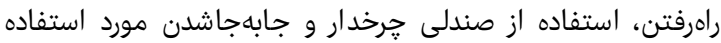

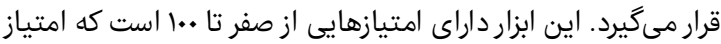

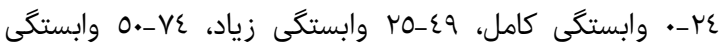

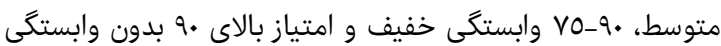

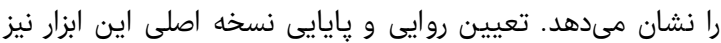
توسط سوليوان و /شميتز [24] انجام شده است فئي

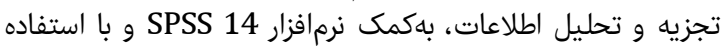

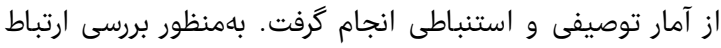

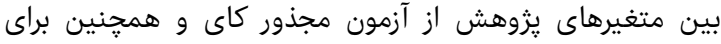

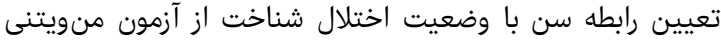

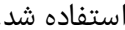

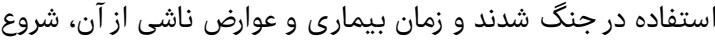

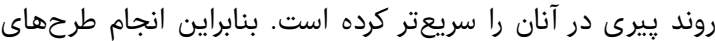

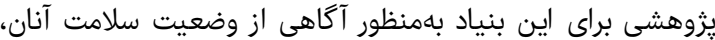

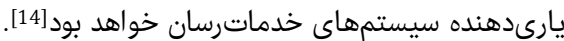

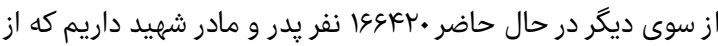

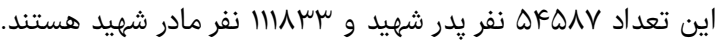

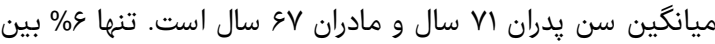

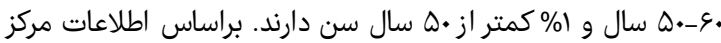

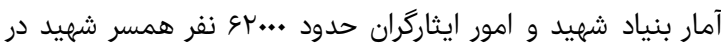

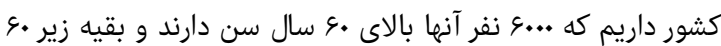

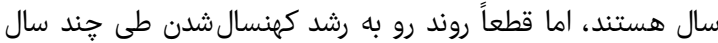

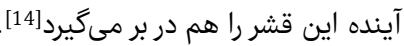

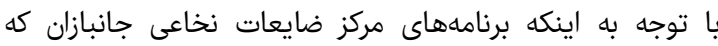

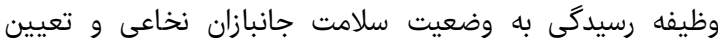

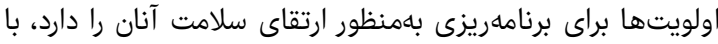

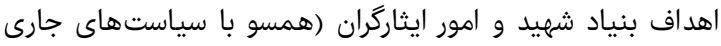

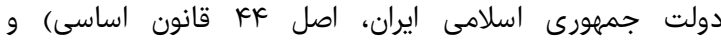

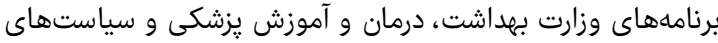

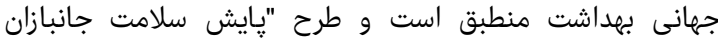

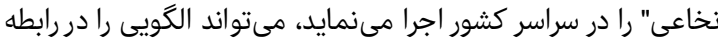

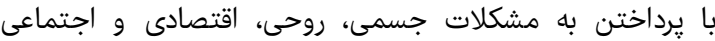

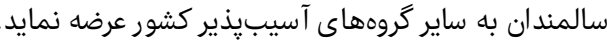

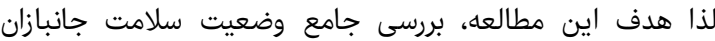

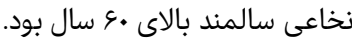

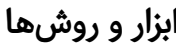

اين تيزوهش توصيفى-مقطعى در سال •وسا در بين كليه افراد

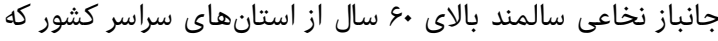

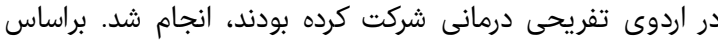

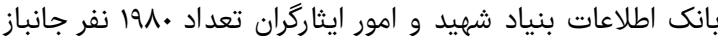

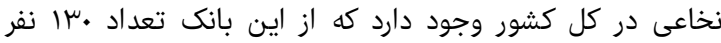

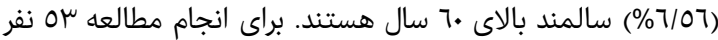

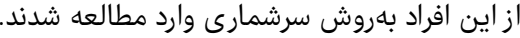
براى تكميل يرسشنامهها، كليه نمونههاى مورد مطالعه قبل از از

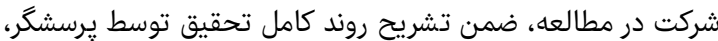

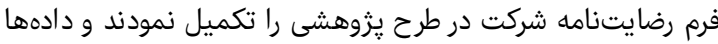

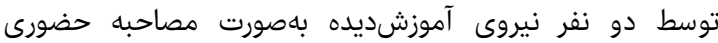
جمع آورى شد.

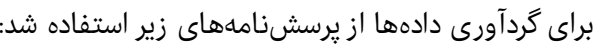

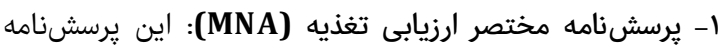

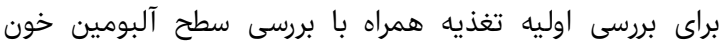

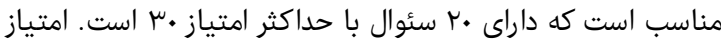

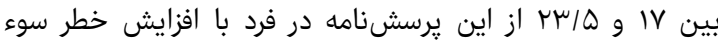

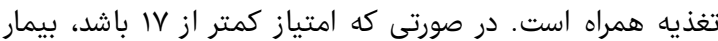

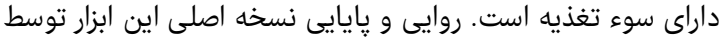

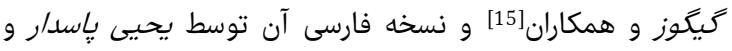
همكاران [16] تاييد شده استارن.

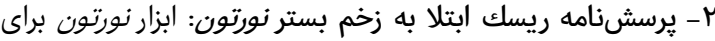

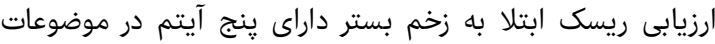

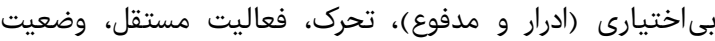


مشكلات سالمندى جانبازان نخاعى و اظهار نظر ييرامون وضعيت

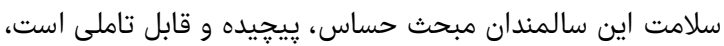

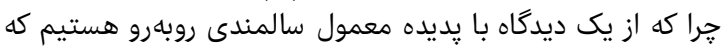

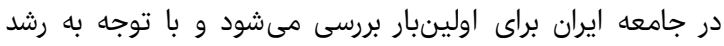

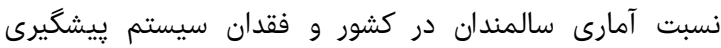

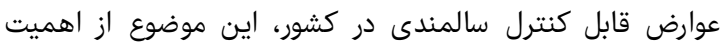

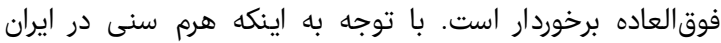

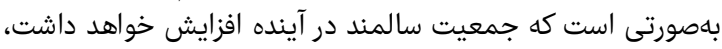

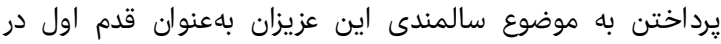

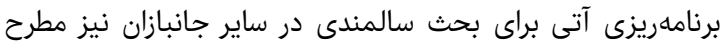

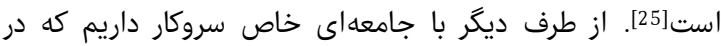

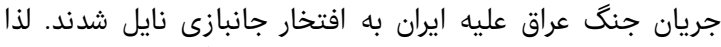

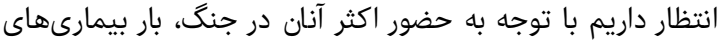

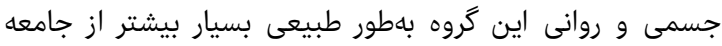

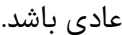
از آنجا كه بيمارىهاى مزمن در سالمندان شامل بيمارىهاى قلبى،

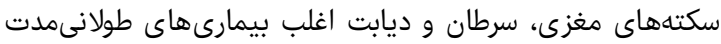

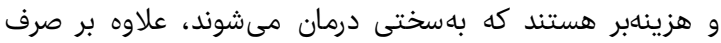

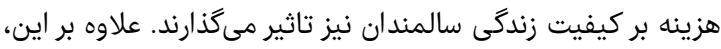

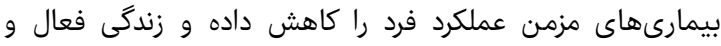

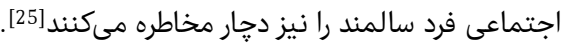

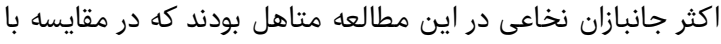

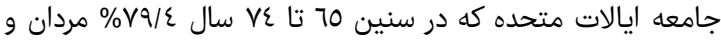

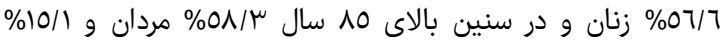

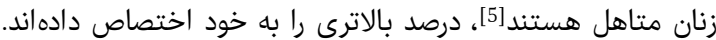

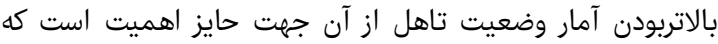

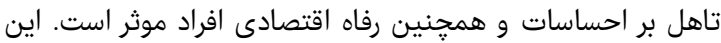

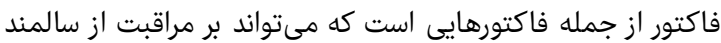

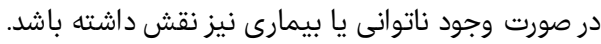

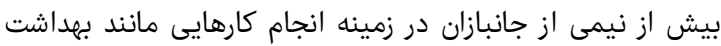

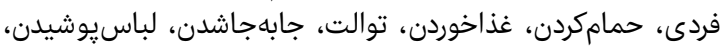

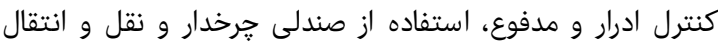

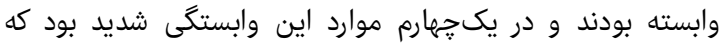

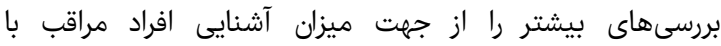

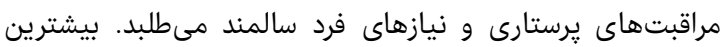

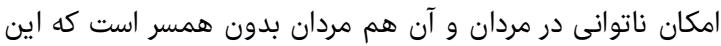

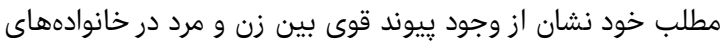

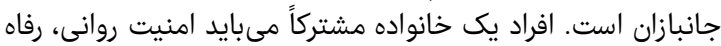

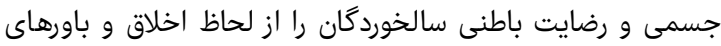

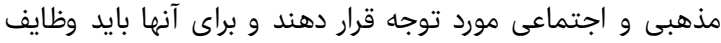

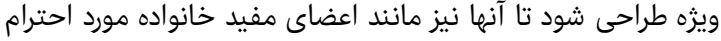
باشند و كار خانوادگى مناسبى به آنها آنها محول شود.

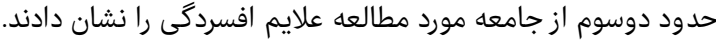

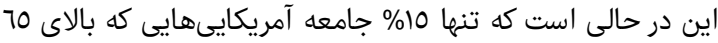
سال سن دارند علايم افسردگى داشتهاند[5]. از آنجا كه سالمندان إندان

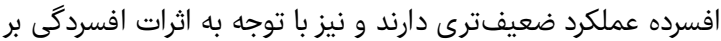

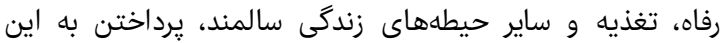

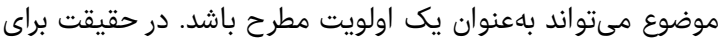

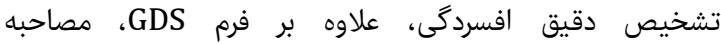

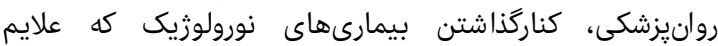

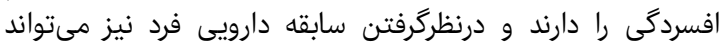

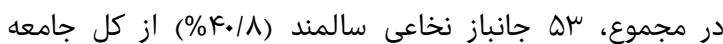

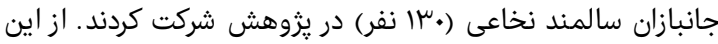

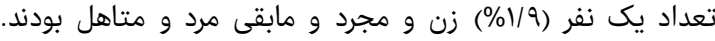

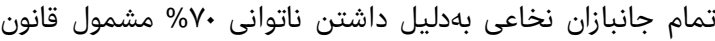

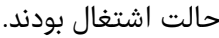

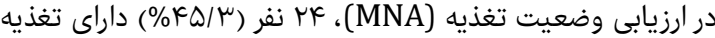

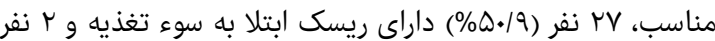

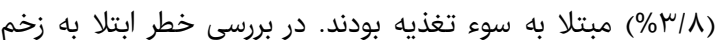

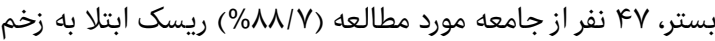

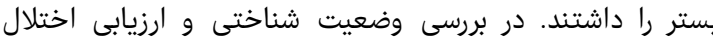

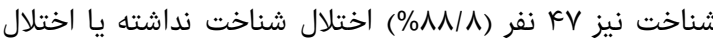

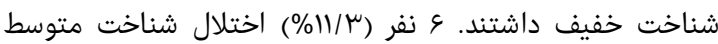

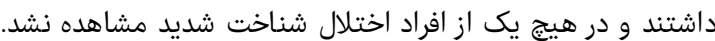

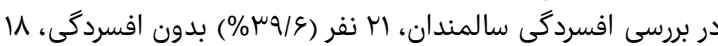

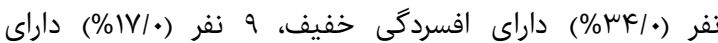

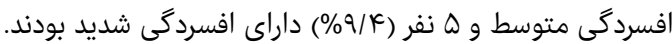

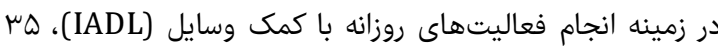

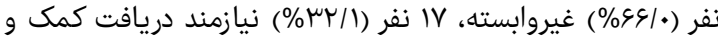

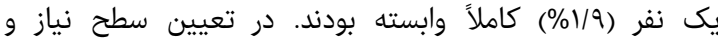

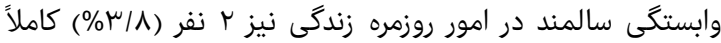

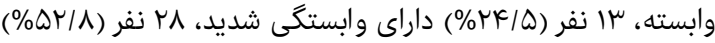

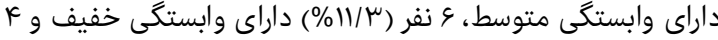

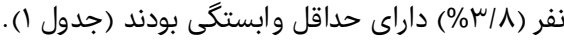

جدول () ارتباط بين وضعيت تغذيه با سطح افسردگى، انجام فعاليتهاى روزانه

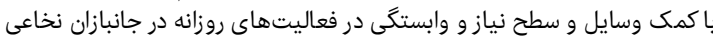
سالمند (رس نفر دراليتها

\begin{tabular}{|c|c|c|c|c|}
\hline كل & داراس تغذيه & سوء ريغذيه & داراى سوء & 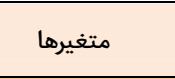 \\
\hline & & & & سطح افسردگى \\
\hline rI & 18 & $\Delta$ & . & عدم افسردگى \\
\hline 11 & $\Delta$ & $I^{\mu}$ & . & فسردگى خفيف \\
\hline 9 & $\mu$ & \& & . & فسردگى متوسط \\
\hline$\Delta$ & • & $\mu$ & r & فسردگى شديد \\
\hline$\Delta \mu$ & rk & rV & r & 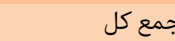 \\
\hline \multicolumn{5}{|c|}{ انجام فعاليتهاى روزانه با كمك وسايل } \\
\hline 1 & . & 1 & . & 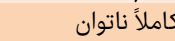 \\
\hline iV & $\mu$ & ir & r & وانايى متوسط \\
\hline ro & r & If & • & 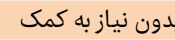 \\
\hline or & rk & rV & r & 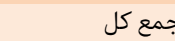 \\
\hline \multicolumn{5}{|c|}{ سطح نياز و وابستكى در فعاليتهاى روزانه } \\
\hline r & 1 & 1 & • & كاملاً وابسته \\
\hline ir & r & 1. & 1 & وابستخى شديد \\
\hline rA & ir & 18 & • & و ابستخى متوسط \\
\hline 7 & $\Delta$ & • & 1 & 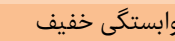 \\
\hline$\varepsilon$ & $k$ & · & . & بدون وابستگى \\
\hline or & KF & rV & r & جمع كل \\
\hline
\end{tabular}

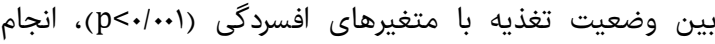

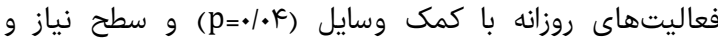

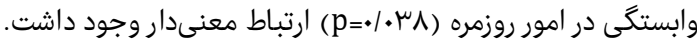
همجنين با ضريب اطمينان 90\% بين سن در جانبازان آردان نخاعى

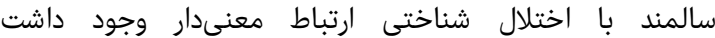

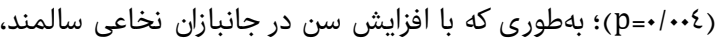

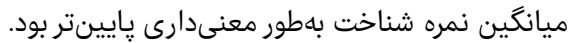
فصل نامه علمى - يزووهشى طب جانباز 
در اين مطالعه خطر ابتلا به زخم بستر جانبازان نخاعى سالمند

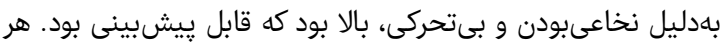

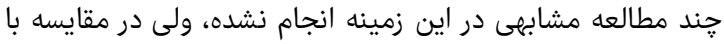

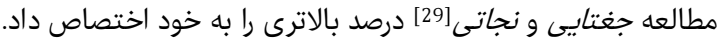

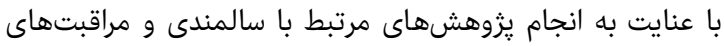

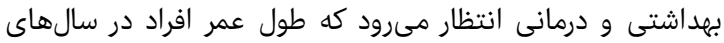

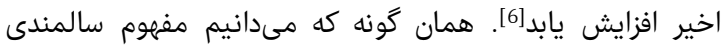

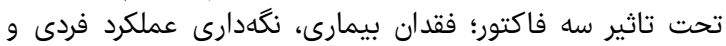

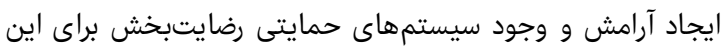
افراد است[30].

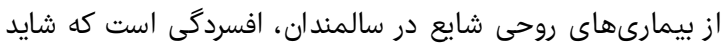

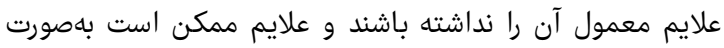

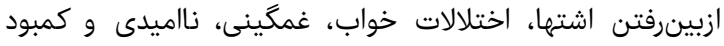

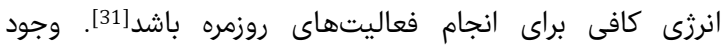

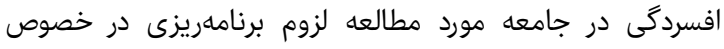

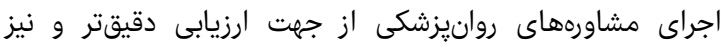

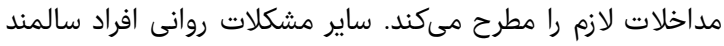

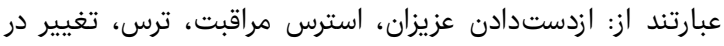

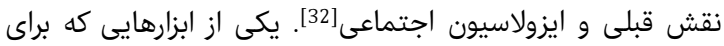

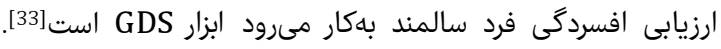

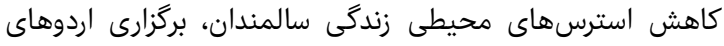

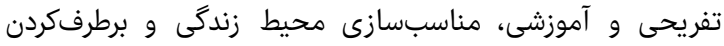

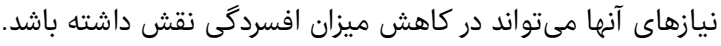

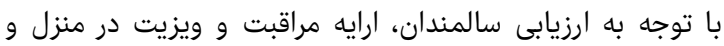

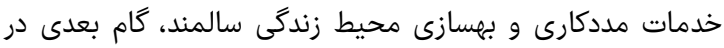

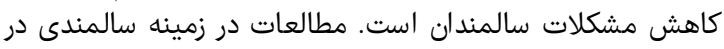

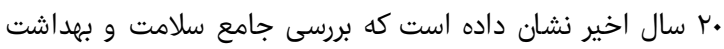

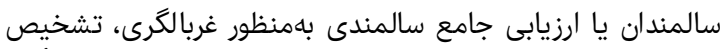

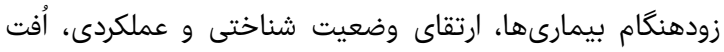

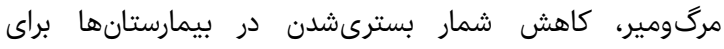

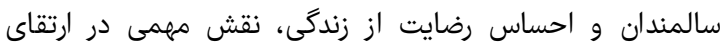

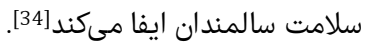

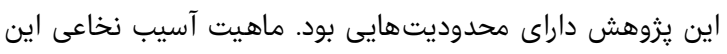

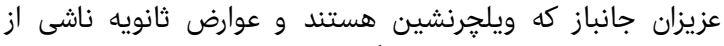

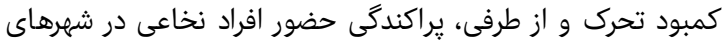

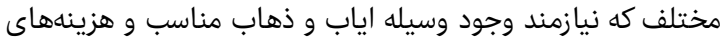

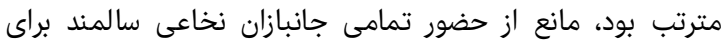

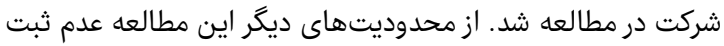

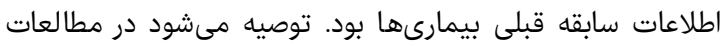

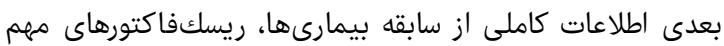

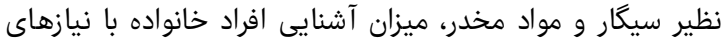

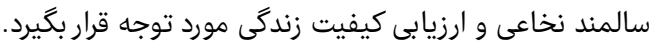

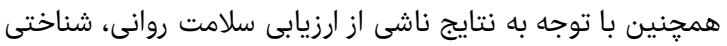

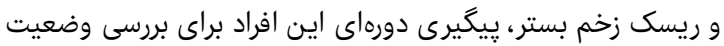

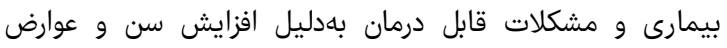
سالمندى بايد انجام شود. بهكاركيرى متخصص بايد انجام تغذيه در كلينيكهاى طب سالمندان بنياد

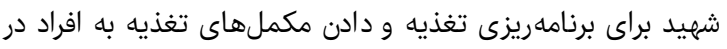

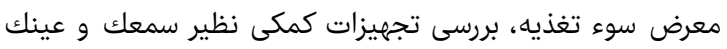

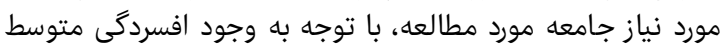

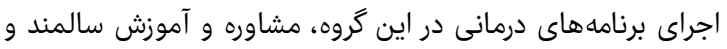

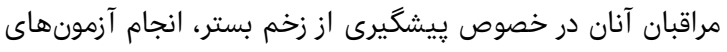

بهعنوان يك اصل مطرح باشد كه استفاده از روشهاى درمانى و

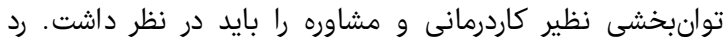

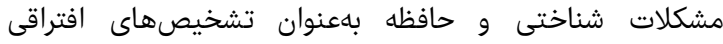

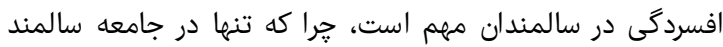

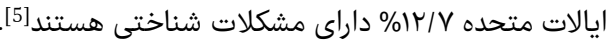

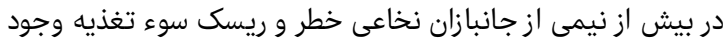

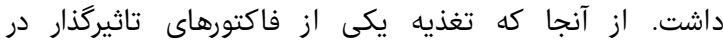

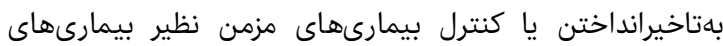

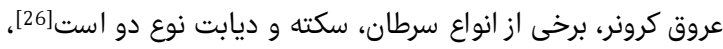

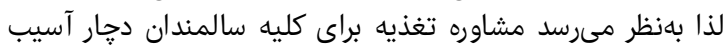

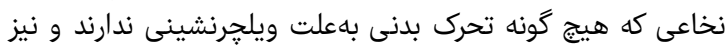

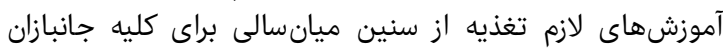

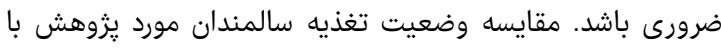

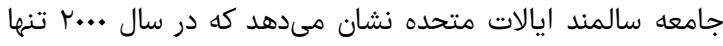

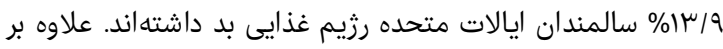

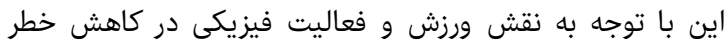

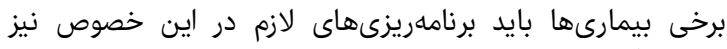

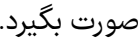

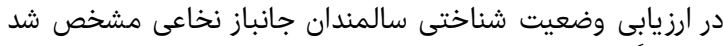

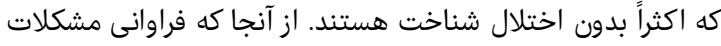

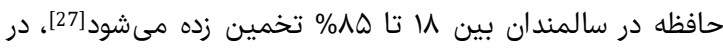

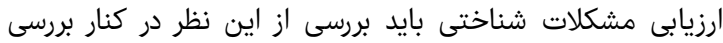

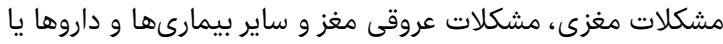

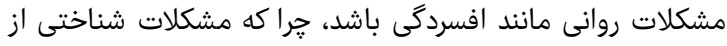

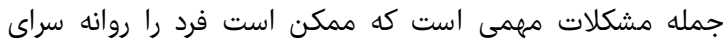
سالمندان كند.

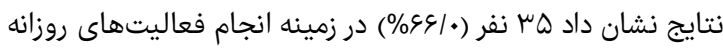

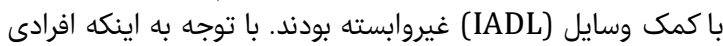

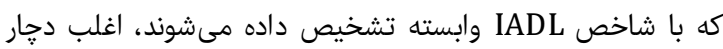

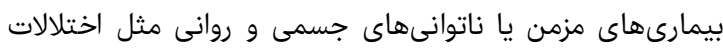

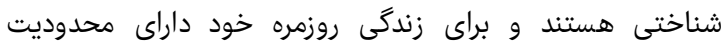

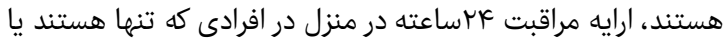

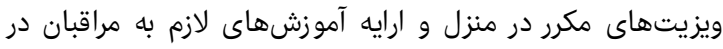

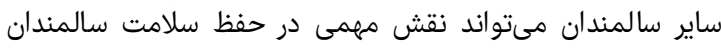

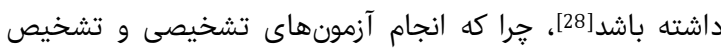

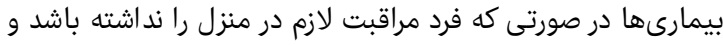

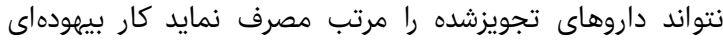

است.

با وجود اينكه در مطالعه حاضر از سابقه بيمارىها، سابقه دارويى،

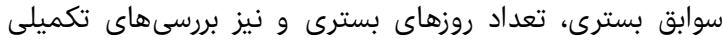
انجامشده قبلى در خصوص مشكلات مطرحشده استفاده نشده، اما

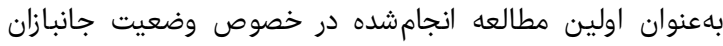

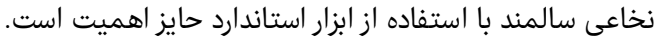

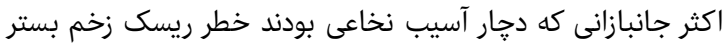

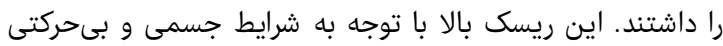

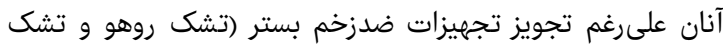

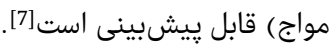

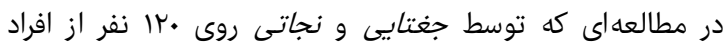

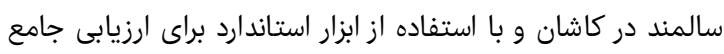

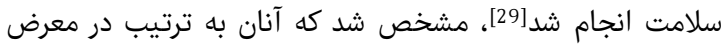

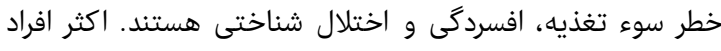
تحت مطالعه در انجام امور روزمره وابسته بودئ لودند 
6- Ingram CR, Phegan KJ, Blumenthal HT. Significance of an aging-linked neuron binding gamma globulin fraction of human sera1. J Gerontol. 1974;29(1):20-7.

7- Nejaty V, Ashayeri H. To investigate the relationship between cognitive dysfunction and depression in the elderly. Salmand. 2006;1(2):112-8. [Persian].

8- Beers MH, Bekow R. The MERCK manual of geriatrics. $3^{\text {rd }}$ edition. Kenilworth, NJ: MERCK; 2000. pp. 48-50.

9- Al-Nasir F, Al-Haddab MK. Levels of disability among the elderly in institutionalized and home-based care in Bahrain. East Mediterr Health J. 1999;5(2):247-54.

10- Butler RN, Lewis MI. Aging and mental health: Positive psychosocial and biomedical approaches. St. Louis: Mosby; 1982.

11- Sorocco KH, McCallum TJ. Mental health promotion in older adults: Addressing treatment approaches and available screening tools. Geriatrics. 2006;61(1):19-22.

12- McColl MA, Charlifue S, Glass C, Savic G, Meehan M. International differences in ageing and spinal cord injury. Spinal Cord .2002;40(3):128-36.

13- Najarian P. Adjustment in the family and patterns of family living. J Soc Issues. 1959;15(3):28-44.

14- Center for Information and Technology. Statistics of Oppressed and War Veterans of the Islamic Revolution Foundation, 2001-2002. Available from: http://www.isaar.ir/vsdgp,9eki5arjar! 9pre5.4r.html

15- Guigoz Y, Vellas B, Garry PJ. Assessing the nutritional status of the elderly: The Mini Nutritional Assessment as part of the geriatric evaluation. Nutr Rev. 1996;54(1 Pt 2):S59-65.

16- Pasdar Y, Qraratappeh A, Pashaee T, Alghasi Sh, Niazi $\mathrm{P}$, Haghnazari L. nutritional status using multidimensional assessment in elderly Iranian. J Kermanshah Univ Med Sci. 2011;15(3):178-86. [Persian] 17- Norton D, McLaren R, Exton-Smith N. An investigation of geriatric nursing problems in hospital. Public Health. 1963;77(5):317.

18- Boban M, Malojčić B, Mimica N, Vuković S, Zrilić I, Hof $\mathrm{PR}$, et al. The reliability and validity of the mini-mental state examination in the elderly Croatian population. Dement Geriatr Cogn Disord. 2012;33(6):385-92.

19- Seyedian M, Falah M, Nourouzian M, Nejat S, Delavar A, Ghasemzadeh HA. Validity of the farsi version of minimental state examination. J Med Counc Islamic Repub Iran. 2008;25(4):414-08. [Persian]

20- Yesavage JA, Brink TL, Rose TL, Lum O, Huang V, Adey M, et al. Development and validation of a geriatric depression screening scale: A preliminary report. J Psychiatr Res. 1982-1983;17(1):37-49.

21- Malakouti SK, Pachana NA, Naji B, Kahani S, Saeedkhani M. Reliability, validity and factor structure of the CES-D in Iranian elderly. Asian J Psychiatr. 2015;18:86-90.

22- Lawton MP, Brody EM. Assessment of older people: Self-monitoring and instrumental activities of daily living measure. Gerontologist. 1969;9(3):179-86.

23- Hassani Mehraban A, Soltanmohamadi Y, Akbarfahimi M, Taghizadeh G. Validity and reliability of the persian version of lawton instrumental activities of daily living scale in patients with dementia. Med J Islam Repub Iran. 2014;28:25.

24- O'Sullivan SB, Schmitz TJ. Physical rehabilitation. $5^{\text {th }}$ edition. Philadelphia: F.A. Davis Company; 2007. p. 385

25- Noroozian M. The elderly population in Iran: An ever growing concern in the health system. Iran J Psychiatry Behav Sci. 2012;6(2):1-6
غربالكرى در سالمندان براى تشخيص سران سريعتر بيمارىها و وانجام برام

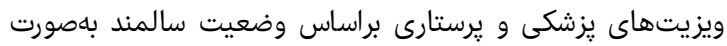

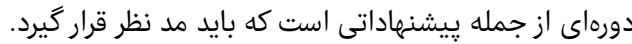

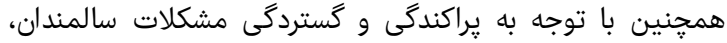

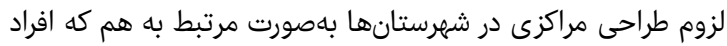

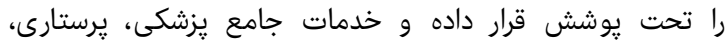

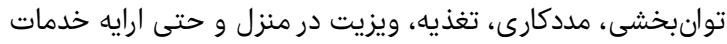

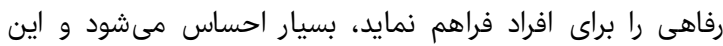

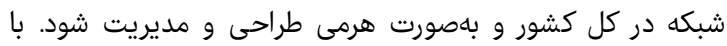
توجه به ارزيابى كلى سلامت سالمند، لزوم حمايك ورايت از اين اين عزيزان

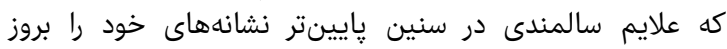
خواهند داد بيش از ييش بايش سايد انجام گيرد.

نتيجهگيرى اكثر جانبازان نخاعى سالمند بالاى •ع سال، بدون اختلال شناخت،

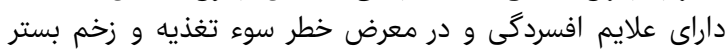

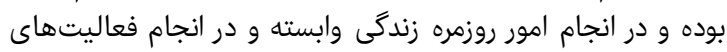

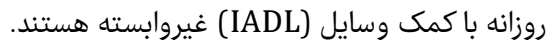

تشكر و قدردانى: از كليه جانبازان معززّ نخاعى سالمند كه با حضور

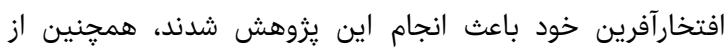

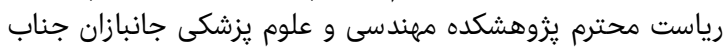

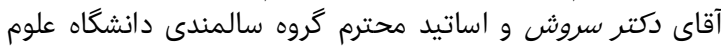

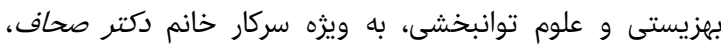
تشكر و قدردانى مى تى تخردد. تاييديه اخلاقى: كليه نمونههاى مورد مرد مطاني مطالعه قبل از شركت در

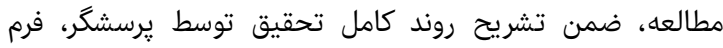

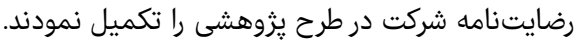

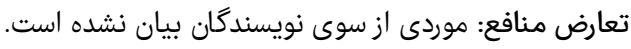

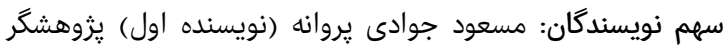

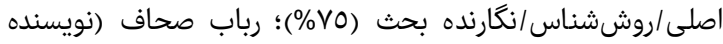

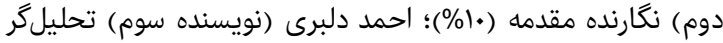

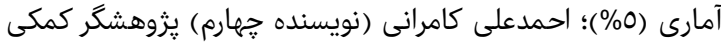

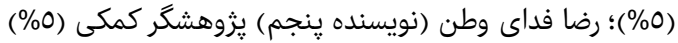

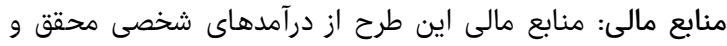

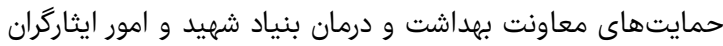
بوده است.

منابع

1- Squire A, Tindal B. Health and wellbeing for older people London. $1^{\text {st }}$ edition. London: Elsevier; 2002. pp. 46.

2- Statistical Center of Iran. General Census of Population and Housing of 2011. Available from: https://www.amar.org.ir/Portals/0/sarshomari90/n_sa rshomari90_2.pdf

3- Shoaee F, Azkhosh M, Alizad V. Health status of iranian older people: A demographical analysis. Salmand. 2013;8(2):60-9. [Persian]

4- Youssef RM. Comprehensive health assessment of senior citizens in Al-Karak governorate, Jordan. East Mediterr Health J. 2005;11(3):334-48.

5- Lewis C, Bottomley J. Demographic of aging in Geriatric Rehabilitation. $3^{\text {rd }}$ edition. New Jersey: Prentice Hall; 2006. 
1.r وضعيت سلامت جانبازان نخاعى سالمند 30 - Kennie DC. Health maintenance of the elderly. Clin
30Geriatr Med. 1986;2(1):53-83.

31- S Stuck AE, Siu AL, Wieland GD, Adams J, Rubenstein LZ. Comprehensive geriatric assessment: A metaanalysis of controlled trials. Lancet. 1993;342(8878):1032-6.

32- Garrett JE. Multiple losses in older adults. J Gerontol Nurs. 1987;13(8):8-12

33- Sheikh JI, Yesavage JA. Geriatric depression scale (GDS) recent evidence and development of a shorter version. Clin Gerontol. 1986;5(1-2):165-72.

34- Fernandez LS, Byard D, Lin CC, Benson S, Barbera JA. Frail elderly as disaster victims: Emergency management strategies. Prehosp Disaster Med. 2002;17(2):67-74.
26- Roberts SB, Rosenberg I. Nutrition and aging: changes in the regulation of energy metabolism with aging. Physiol Rev. 2006;86(2):651-67.

27- Walker PC, Alrawi A, Mitchell JF, Regal RE, Khanderia U. Medication use as a risk factor for falls among hospitalized elderly patients. Am J Health Syst Pharm. 2005;62(23):2495-9.

28- Halter JB, Ouslander JG, Studenski S, High KP, Asthana S, Supiano MA, et al. Hazzard's geriatric medicine and gerontology. $7^{\text {th }}$ edition. New York: McGraw-Hill Professional; 2009.

29- Joghataei MT, Nejati V. Assessment of health status of elderly people in the city of Kashan. Salmand. 2006;1(1):3-10. [Persian] 